\title{
miR-142 inhibits the migration and invasion of glioma by targeting Rac1
}

\author{
WENYI QIN ${ }^{1}$, XIAOFENG RONG ${ }^{1}$, JIANGCHUAN DONG ${ }^{2}$, CHAO YU $^{3}$ and JUAN YANG ${ }^{1}$ \\ ${ }^{1}$ Department of Integrated Chinese and Western Medicine, The First Affiliated Hospital of Chongqing Medical University, \\ Chongqing 400016; ${ }^{2}$ Department of Emergency, The Second Affiliated Hospital of Chongqing Medical University, \\ Chongqing 400010; ${ }^{3}$ Department of Emergency, Chongqing JiangBei Hospital of \\ Traditional Chinese Medicine, Chongqing 400020, P.R. China
}

Received November 23, 2016; Accepted June 16, 2017

DOI: 10.3892/or.2017.5816

\begin{abstract}
Increasing evidence has shown that aberrant microRNAs (miRNAs) are implicated in tumorigenesis and tumor progression by regulating oncogenes or tumor suppressors. Dysregulation of miR-142 has been reported in multiple tumors. However, its clinical roles and underlying mechanism in glioma remain to be elucidated. In the present study, we found that the expression of miR-142 was significantly downregulated in both glioma tissues and cell lines by qRT-PCR. Clinical analysis revealed that decreased miR-142 was markedly associated with advanced World Health Organization (WHO) grade. Moreover, we disclosed that miR-142 was a novel independent prognostic marker in the prediction of the 5-year survival of glioma patients. The ectopic overexpression of miR-142 inhibited cell migration, invasion and invasion-related gene expression. Notably, miR-142 modulated Rac1 by directly binding to its 3'-untranslated (3'-UTR) region, leading to the suppression of the expression of matrix metalloproteinases (MMPs). In glioma clinical samples, miR-142 was inversely correlated with Rac1 expression, and played positive roles in glioma migration and invasion. Alteration of Rac1 expression at least partially abolished the migration, invasion and MMP expression of miR-142 in glioma cells. In the present study, we identified Racl as a functional target of miR-142 in glioma. In conclusion, our data indicated that miR-142 inhibited the migration, invasion and MMP expression of glioma by targeting Racl, and may represent a novel potential therapeutic target and prognostic marker for glioma.
\end{abstract}

Correspondence to: Professor Juan Yang, Department of Integrated Chinese and Western Medicine, The First Affiliated Hospital of Chongqing Medical University, 1 Youyi Road, Yuzhong, Chongqing 400016, P.R. China

E-mail: yangj0531@126.com

Key words: microRNA-142, glioma, Rac1, MMP, invasion

\section{Introduction}

Glioma is the most common, aggressive and lethal type of malignant tumor of the central nervous system $(1,2)$. Its relapse and mortality rate are constantly increasing due to the inefficiency of current treatment and this has led to its poor prognosis despite the combination of multidisciplinary therapies including surgical resection, chemotherapy and radiotherapy $(3,4)$. Although diverse therapeutic efforts have been made, the median survival of glioma patients has not changed markedly in the last few years $(5,6)$. The poor prognosis highlights the urgent need to elucidate the detailed molecular mechanism for the development of novel therapeutic tools against glioma and the identification of diagnostic and prognostic markers of glioma (7).

MicroRNAs (miRNAs) are an endogenous group of evolutionarily conserved, non-coding RNAs, which function as negative modulators of gene expression at the posttranscriptional level via binding to the $3^{\prime}$-untranslated (3'-UTR) regions of target mRNAs through complementary binding, causing translational suppression or mRNA degradation (8-10). Accumulating evidence has confirmed that miRNAs are involved in diverse fundamental biological processes including cell differentiation, apoptosis, proliferation and invasion (11-13). An increasing number of studies have demonstrated that aberrant miRNAs play crucial roles in cancer pathogenesis and progression (14). Recently, miR-142 was reported to be dysregulated in multiple cancers (15-17). For instance, it was significantly downregulated in cervical cancer (13), osteosarcoma (18), lung (19) and colon cancer $(20,21)$. Previous studies revealed that miR-142 was identified as a regulator of cell proliferation, migration and invasion in different cancers. miR-142 inhibits cell proliferation and invasion of cervical cancer cells by targeting FZD7 (13). miR-142 functions as a potential tumor suppressor in human osteosarcoma by targeting HMGA1 (22). In addition, serum miR-142 is associated with early relapse in operable lung adenocarcinoma patients (23). However, the expression of miR-142 is overexpressed in nasopharyngeal carcinoma (24), testicular (25), and head and neck cancer (26), esophageal squamous cell (27) and renal cell carcinoma (28). miR-142 suppresses SOCS6 expression and promotes cell proliferation 
in nasopharyngeal carcinoma (24). Oncogenic miR-142 is associated with cellular migration, proliferation and apoptosis in renal cell carcinoma (28). Therefore, it is suggested that the functional significance of miR-142 in tumorigenesis and progression is cancer-type specific. However, the functional role and the underlying molecular mechanism by which miR-142 regulates the initiation and development of glioma have yet to be elucidated.

In the present study, we investigated the expression and biological function of miR-142 in glioma progression. We found that miR-142 was significantly decreased in both glioma tissues and cell lines. Its expression level was closely correlated with World Health Organization (WHO) grading and poor survival outcome in patients. miR-142 inhibited glioma cell migration and invasion in vitro as determined by gain- and loss-of-function experiments by directly targeting Racl thus leading to the suppression of matrix metalloproteinases (MMPs). Collectively, these data confirmed the underlying mechanism by which miR-142 inhibits migration and invasion of glioma and identified miR-142 as a novel prognostic biomarker for glioma patients.

\section{Materials and methods}

Clinical tissues and cell lines. Ninety-seven glioma and 25 human normal brain tissues were obtained from The First Affiliated Hospital of Chongqing Medical University from January 2007 to December 2010. None of the patients had received local or systemic treatment before surgery. Informed consent was obtained from all the patients and the present study was approved by the Ethics Committee of the First Affiliated Hospital of Chongqing Medical University according to the Declaration of Helsinki.

Human glioma cell lines (U87, U251, LN229 and U373) and normal human astrocytes (NHA) were purchased from the Institute of Biochemistry and Cell Biology (Chinese Academy of Sciences, Shanghai, China) and were cultured in Dulbecco's modified Eagle's medium/F12 (DMEM; HyClone, Logan, UT, USA) supplemented with $10 \%$ fetal bovine serum (FBS; Gibco, Grand Island, NY, USA) and 1\% penicillin/streptomycin (Sigma-Aldrich, St. Louis, MO, USA) at $37^{\circ} \mathrm{C}$ with $5 \%$ $\mathrm{CO}_{2}$.

RNA extraction, quantitative real-time PCR ( $q R T-P C R)$. Total RNA was extracted from tissues and cells by TRIzol reagent (Invitrogen, Carlsbad, CA, USA) according to the manufacturer's instructions. RNA was reverse-transcribed using a TaqMan Human MiRNA Assay kit (Applied Biosystems, Foster City, CA, USA) and cDNA was then amplified with a SYBR ${ }^{\circledR}$ Premix Ex Taq ${ }^{\mathrm{TM}}$ II (Perfect RealTime) kit (Takara Bio Inc., Shiga, Japan) and analyzed using an Applied Biosystems 7900 Real-Time PCR System. Hsa-miR-142 primer (HmiRQP0185), snRNA U6 qPCR primer (HmiRQP9001) and GAPDH (HQP006940) were purchased from GeneCopoeia (Guangzhou, China). The primer for Rac1 was synthesized by GenePharma (Shanghai, China).

Western blotting. Proteins were isolated in RIPA buffer (Beyotime, Haimen, China) and the protein concentration was determined using a Bicinchoninic Acid (BCA) Protein Assay kit (Thermo Fischer Scientific, Rockford, IL, USA). Protein $(40 \mu \mathrm{g})$ was separated on $10 \%$ SDS-PAGE and transferred to polyvinylidene difluoride (PVDF) membranes (Millipore, Billerica, MA, USA). The membranes were blocked with 5\% non-fat milk in Tris-buffered saline with Tween-20 (TBST) and then incubated at $4^{\circ} \mathrm{C}$ overnight with primary antibodies: Rac1, MMP2, MMP9 and GAPDH (1:1,000; Cell Signaling Technology, Inc., Danvers, MA, USA). After being washed with TBST, the membranes were incubated with an appropriate peroxidase-conjugated secondary antibody for $2 \mathrm{~h}$ at room temperature (ZSGB-BIO, Beijing, China). Protein bands were visualized using an enhanced chemiluminescence kit (Amersham, Little Chalfont, UK).

Immunohistochemical staining analysis (IHC). IHC was performed on formalin-fixed paraffin sections. MMP2 and MMP9 (Cell Signaling Technology, Inc.) (1:100) antibodies were used in immunohistochemical staining with the streptavidin peroxidase-conjugated (SP-IHC) method. The percentage of positive cells was expressed as the following grades: $0,<5 \%$; $1,6-25 \% ; 2,26-50 \% ; 3,51-75 \%$; and $4,>75 \%$.

Cell transfection. The cells were seeded into 6-well plates and transfected with vectors using Lipofectamine 2000 reagent (Invitrogen Life Technologies) in accordance with the manufacturer's protocol. miRNA expression vectors, including miR-142 expression vector (HmiR0282), the control vector (CmiR0001-MR04) were obtained from GeneCopoeia. The Rac1 expression plasmid was produced by GenePharma.

Wound-healing migration assay. To confirm the migratory ability of glioma cells, we performed a wound-healing assay in each group. Cells were seeded into 6-well plates and when confluence reached $80-90 \%$, a $200 \mu \mathrm{l}$ tip was used to create a wound across the cell plates. Subsequently the cells were washed twice with phosphate-buffered saline (PBS) to remove the detached cells. The cells were then cultured in DMEM in a common incubator for $48 \mathrm{~h}$. Finally, the images were captured using phase-contrast microscope.

Transwell invasion assays. Cell invasion assays were determined by $8-\mu \mathrm{M}$ pore-sized Transwell inserts (Nalge Nunc International Corp., Naperville, IL, USA). The upper chambers were pre-coated with Matrigel (BD Biosciences, Franklin Lakes, NJ, USA). The cells $\left(2.5 \times 10^{4}\right)$ in $150 \mu \mathrm{l}$ of serum-free DMEM were loaded into the top chamber, and $750 \mu \mathrm{l}$ of DMEM with $10 \%$ FBS were placed in the bottom chamber. After $24 \mathrm{~h}$ of incubation, the cells were fixed in $4 \%$ paraformaldehyde and stained with $0.3 \%$ crystal violet. The cells remaining in the top layer were swabbed carefully and the bottom cells were counted under microscope.

Luciferase reporter assay. Luciferase activity was determined using Dual Luciferase Assay (Promega, Madison, WI, USA) according to the manufacturer's protocols. The wild-type 3'-UTR of Racl containing the putative miR-142 target site was amplified and cloned into the pGL3-control luciferase reporter vector (Promega). The mutant constructs were generated using a QuickChange site-directed mutagenesis kit (Stratagene, 

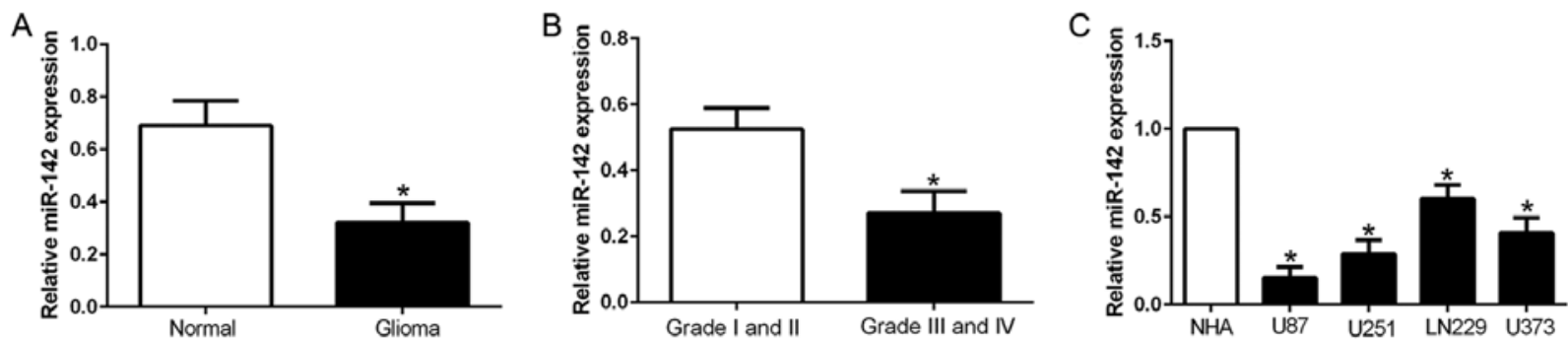

Figure 1. Decreased expression of miR-142 in human glioma tissues and cells. (A) qRT-PCR analysis was performed. The results revealed that the expression of miR-142 expression in glioma tissues was significantly lower than that in normal brain tissues. (B) The expression of miR-142 was lower in high grade glioma tissues than in low grade glioma. (C) Comparison of the expression levels of miR-142 between different glioma cell lines compared to normal human astrocytes. $\mathrm{n}=$ six repeats with similar results. U6 snRNA was used as an internal control; ${ }^{*} \mathrm{P}<0.05$.

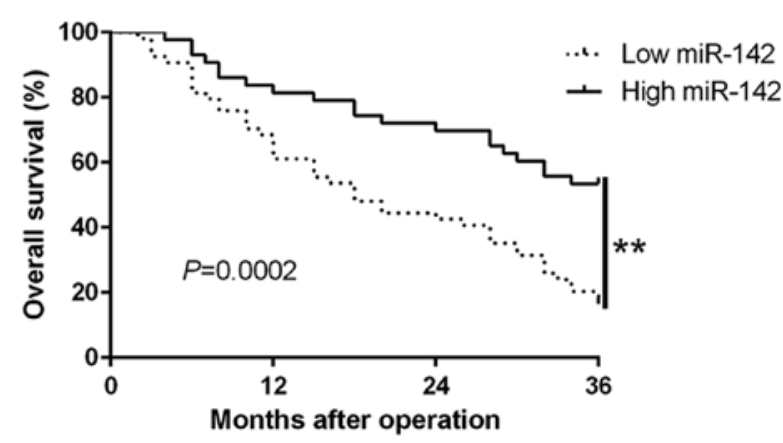

Figure 2. Prognostic value of miR-142 in glioma patients. Glioma patients with low expression of miR-142 had poorer overall survival; ${ }^{* *} \mathrm{P}<0.01$.

La Jolla, CA, USA). The experiment was performed in duplicate in three independent experiments.

Statistical analysis. Data are presented as the mean \pm SD from at least three independent replicates. SPSS software 16.0 (SPSS, Inc., Chicago, IL, USA) and GraphPad Prism 6.0 (GraphPad, San Diego, CA, USA CA, USA) were used for a two-tailed Student's t-test, Pearson's correlation analysis, Kaplan-Meier method and the log-rank test to evaluate statistical significance. Differences were defined as $\mathrm{P}<0.05$.

\section{Results}

Decreased expression of miR-142 in human glioma tissues and cells. We performed qRT-PCR to investigate the expression level of miR-142 in clinical tissues. As shown in Fig. 1A, the expression of miR-142 was significantly decreased in the glioma tissues compared with the normal brain tissues (Fig. 1A; $\mathrm{P}<0.05)$. According to the pathological diagnosis, the expression of miR-142 in the high grade glioma (III and IV) were lower than that in the low grade glioma tissues (I and II), which indicated that miR-142 expression was associated with glioma malignancy (Fig. 1B; $\mathrm{P}<0.05$ ). Consistently, all glioma cell lines had significantly decreased expression of miR-142 than normal astrocytes (Fig. $1 \mathrm{C} ; \mathrm{P}<0.05$ ). These data revealed that miR-142 may act as a tumor suppressor in glioma.

Decreased expression of miR-142 predicts a poor prognosis. To assess the clinical significance of miR-142 in glioma, we determined the median expression value as a cut-off to divide glioma patients into groups of high or low expression levels
Table I. Association of miR-142 expression with clinicopathological characteristics of glioma patients.

\begin{tabular}{|c|c|c|c|c|}
\hline \multirow[b]{2}{*}{$\begin{array}{l}\text { Clinical } \\
\text { parameters }\end{array}$} & \multirow[b]{2}{*}{$\begin{array}{l}\text { Cases } \\
\text { (n) }\end{array}$} & \multicolumn{2}{|c|}{$\begin{array}{c}\text { miR-142 } \\
\text { expression level }\end{array}$} & \multirow[b]{2}{*}{$\begin{array}{l}\text { P-value } \\
\left({ }^{\mathrm{a}} \mathrm{P}<0.05\right)\end{array}$} \\
\hline & & $\begin{array}{l}\text { High } \\
(n=43)\end{array}$ & $\begin{array}{c}\text { Low } \\
(n=54)\end{array}$ & \\
\hline Age (years) & & & & 0.936 \\
\hline$<60$ & 65 & 29 & 36 & \\
\hline$\geq 60$ & 32 & 14 & 18 & \\
\hline Sex & & & & 0.629 \\
\hline Male & 59 & 25 & 34 & \\
\hline Female & 38 & 18 & 20 & \\
\hline WHO grade & & & & $0.001^{\mathrm{a}}$ \\
\hline $\mathrm{I}+\mathrm{II}$ & 34 & 24 & 10 & \\
\hline III+IV & 63 & 19 & 44 & \\
\hline Location & & & & 0.791 \\
\hline Supratentorial & 69 & 30 & 39 & \\
\hline Subtentorial & 28 & 13 & 15 & \\
\hline
\end{tabular}

miR-142, microRNA-142; WHO, World Health Organization; ${ }^{\text {aSta- }}$ tistically significant.

of miR-142. As shown in Table I, low expression of miR-142 was prominently associated with the histological grade of the glioma $(\mathrm{P}=0.001)$. Hence, these findings revealed that decreased expression of miR-142 was involved in the progression of glioma. Moreover, survival analysis revealed that the downregulation of miR-142 was significantly correlated with shorter overall survival ( $\mathrm{P}=0.0002$; Fig. 2$)$ in glioma patients. Furthermore, miR-142 expression was an independent prognostic factor for predicting overall survival in glioma patients $(\mathrm{P}=0.001 ; 0.001$, respectively; Table II). These results revealed that miR-142 may possibly serve as a potential prognostic biomarker in glioma patients.

miR-142 inhibits cell migration and invasion in human glioma cells. To explore the biological function of miR-142 in glioma, we transfected miR-142 mimics to ectopically express this miRNA in U87 and U251 cells. The results revealed that the expression of miR-142 was significantly increased by appropriate vectors 
Table II. Univariate and multivariate analysis of prognostic factors in glioma patients.

\begin{tabular}{|c|c|c|c|c|c|c|}
\hline \multirow[b]{2}{*}{ Variables } & \multicolumn{3}{|c|}{ Univariate analysis } & \multicolumn{3}{|c|}{ Multivariate analysis } \\
\hline & HR & $95 \% \mathrm{CI}$ & P-value & HR & $95 \% \mathrm{CI}$ & P-value \\
\hline Age (years) & 1.205 & $0.563-2.273$ & 0.458 & 1.180 & $0.397-1.709$ & 0.436 \\
\hline Sex & 1.538 & $0.558-2.783$ & 0.523 & 1.039 & $0.482-1.927$ & 0.329 \\
\hline WHO grade & 4.327 & $1.872-5.674$ & $0.001^{\mathrm{a}}$ & 2.512 & $1.287-3.165$ & $0.002^{\mathrm{a}}$ \\
\hline miR-142 & 3.965 & $1.583-7.382$ & $0.001^{\mathrm{a}}$ & 3.218 & $1.237-6.962$ & $0.001^{\mathrm{a}}$ \\
\hline
\end{tabular}

HR, hazard ratio; CI, confidence interval; miR-142, microRNA-142; WHO, World Health Organization; ${ }^{a}$ Statistically significant.
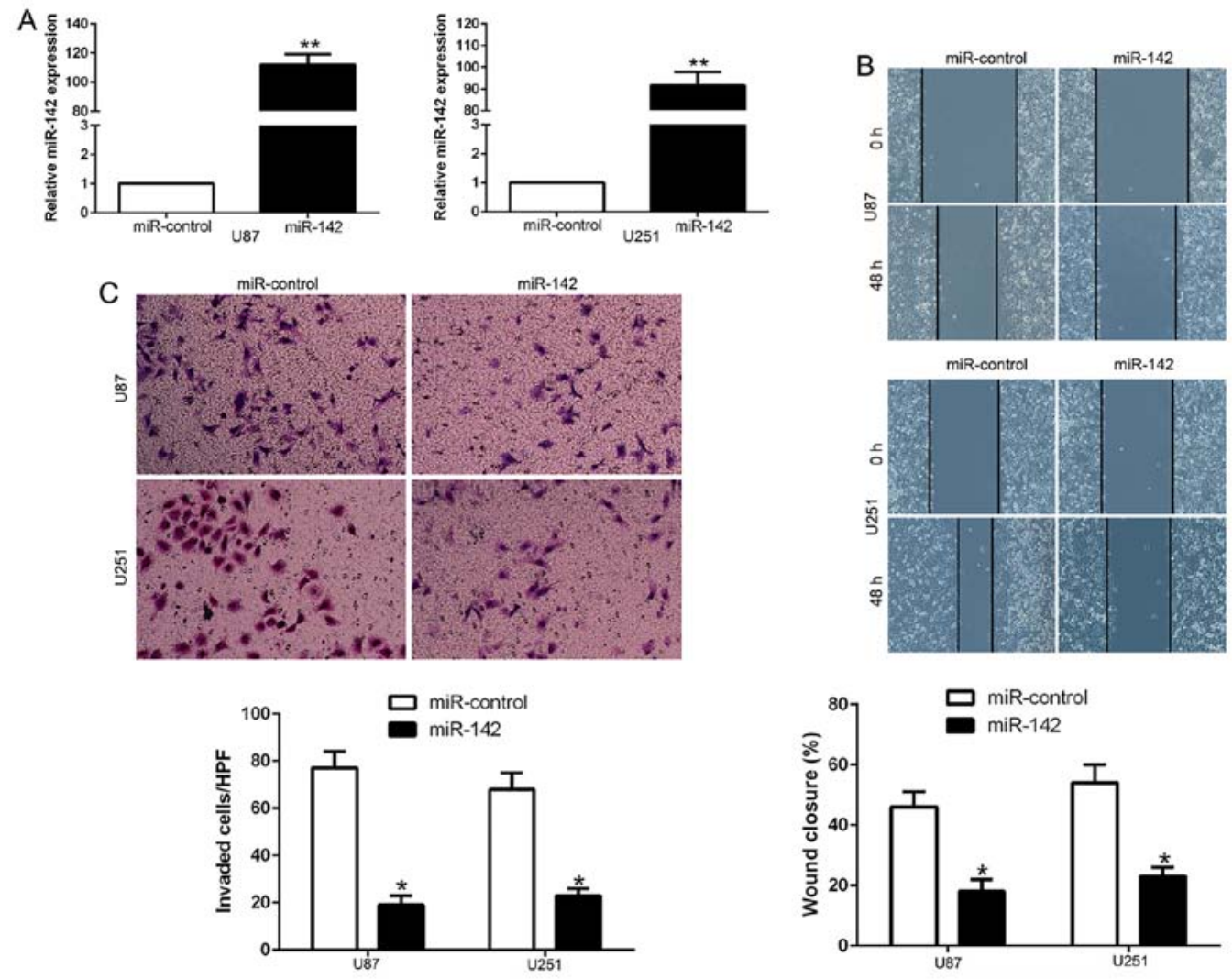

Figure 3. miR-142 inhibits glioma cell migration and invasion in vitro. (A) U87 and U251 cells were transfected with miR-142 mimics or negative control. Twenty-four hours later the cells were subjected to qRT-PCR to determine the expression of miR-142 in U87 and U251 cells. (B) Cell migration as assessed by wound-healing assays was inhibited by overexpression of miR-142 in U87and U251 cells. (C) Cell invasion as assessed by Transwell assays was suppressed by ectopic miR-142 expression in U87 and U251 cells. $\mathrm{n}=$ six independent experiments; $\mathrm{P}<0.05,{ }^{* *} \mathrm{P}<0.01$.

in both cell lines as determined by qRT-PCR (Fig. 3A; $\mathrm{P}<0.01$ ). Wound-healing assay demonstrated that the overexpression of miR-142 significantly inhibited the migration ability of U87 and U251 cells (Fig. 3B; $\mathrm{P}<0.05$ ). Consistently, Transwell assay revealed that the ectopic expression of miR-142 suppressed the invasive number of glioma cells (Fig. $3 \mathrm{C}$; $\mathrm{P}<0.05$ ). These results demonstrated that miR-142 regulates the migration and invasion of glioma cells.

Overexpression of miR-142 inhibits invasion-related genes. MMP2 and MMP9 are important members of the MMP family, which degrade all essential components of the extracellular matrix and play critical roles in cancer metastasis, including glioma. We disclosed whether miR-142 modulated these invasion-related genes. Our results revealed that miR-142 overexpression significantly decreased the expression of MMP2 and MMP9 in both U87 and U251 cells $(\mathrm{P}<0.05$; Fig. 4A and B). Moreover, we investigated the correlation between miR-142 expression and MMPs in glioma tissues. We found that MMP2 and MMP9 expression in the high miR-142 expression group was significantly lower than that in the low expression miR-142 group ( $\mathrm{P}<0.05$; Fig. $4 \mathrm{C})$. In conclusion, these results revealed that miR-142 is critical for glioma cell invasion by regulating the expression of MMPs.

Racl is the direct downstream target of miR-142. We searched for candidate target genes of miR-142 by exploring different algorithm programs (TargetScan and miRanda) and predicted 

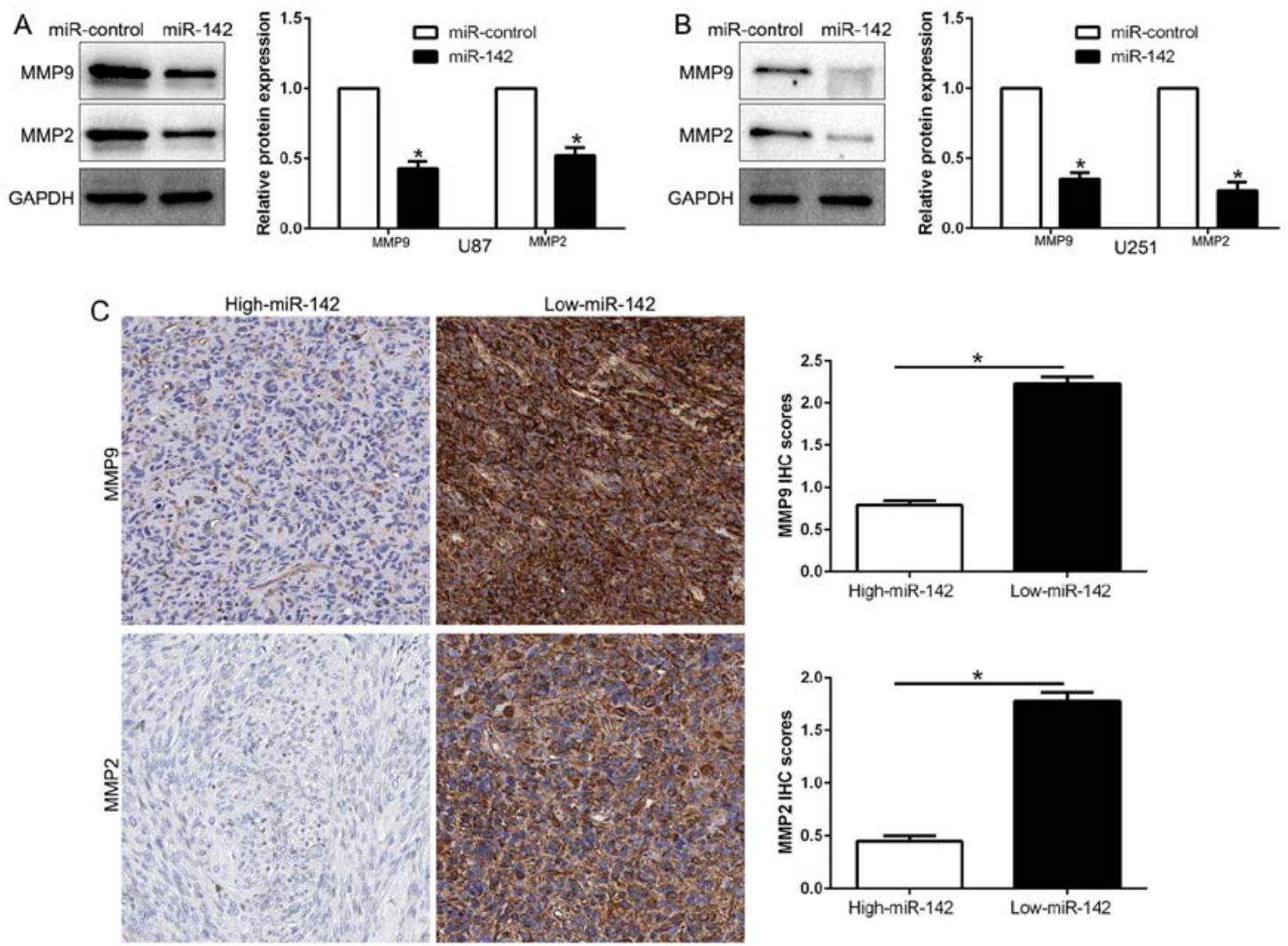

Figure 4. miR-142 inhibits MMP2 and MMP9 expression in glioma cells. The overexpression of miR-142 in (A) U87 and (B) U251 cells decreased the expression MMP2 and MMP9. (C) Immunohistochemical analysis of MMP2 and MMP9 in glioma samples. In cases of low miR-142 expression (right panel); there was strong MMP2 and MMP9 protein expression in the tissue sections. In contrast, in the case of high miR-142 expression (left panel), there was no detectable MMP2 and MMP9 protein expression. Values are depicted as the mean \pm SEM; ${ }^{*} \mathrm{P}<0.05$ by t-test. MMP, matrix metalloproteinase.
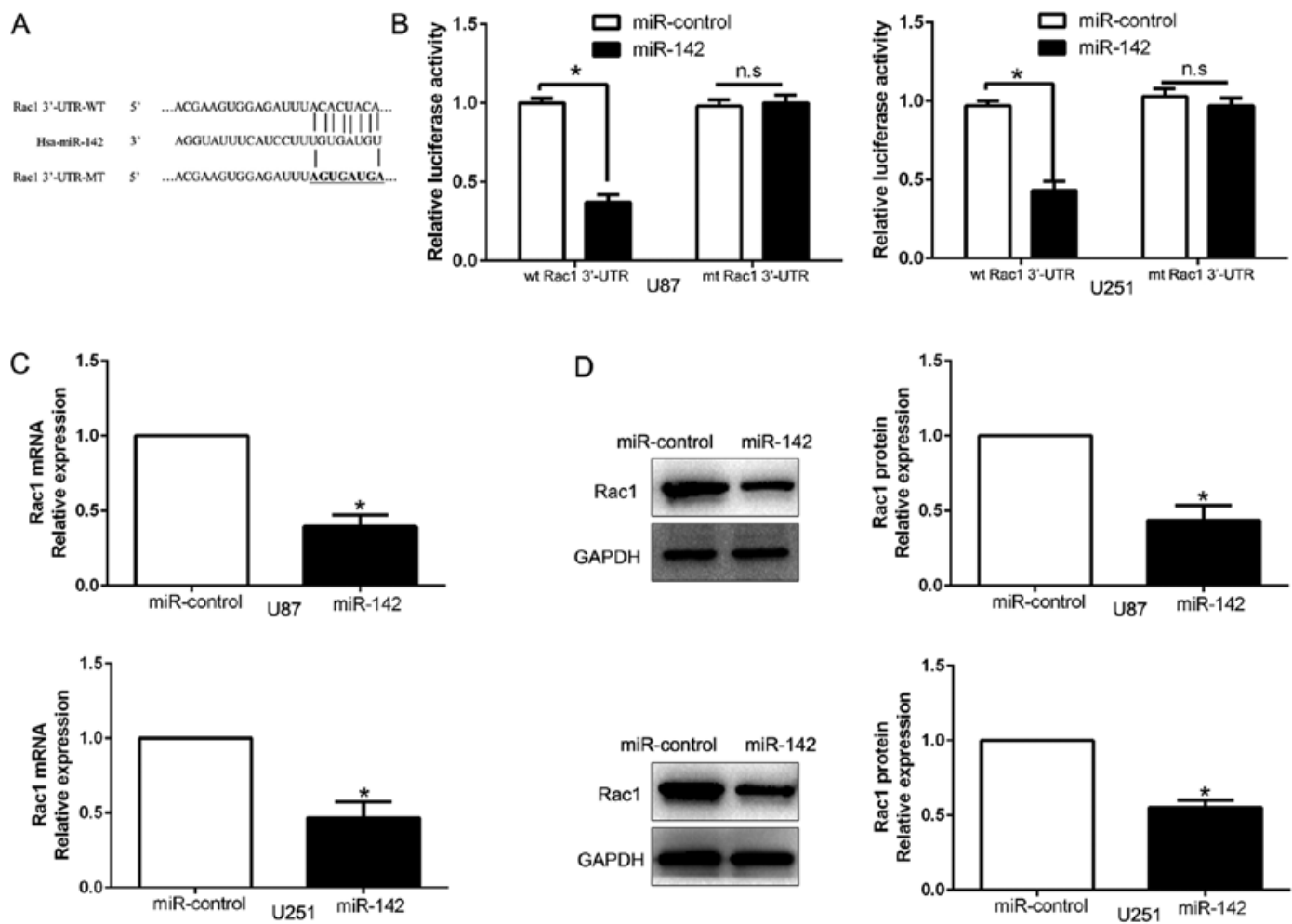

Figure 5. Rac1 is identified as a direct target of miR-142 in glioma. (A) miR-142 and its putative binding sequence in the 3'-UTR of Rac1. The mutant binding site was generated in the complementary site for the seed region of miR-142. (B) miR-142 significantly suppresses the luciferase activity that carried the wild-type (wt), but not the mutant (mt) 3'-UTR of Rac1 in U87 and U251 cells. (C) qRT-PCR analysis of Rac1 mRNA expression in U87 and U251 cells with miR-142 or miR-control vector transfection. (D) Overexpression of miR-142 decreased the expression of Rac1 protein in U87 and U251 cells. n=six repeats with similar results; ${ }^{\mathrm{P}}<0.05$. 
A
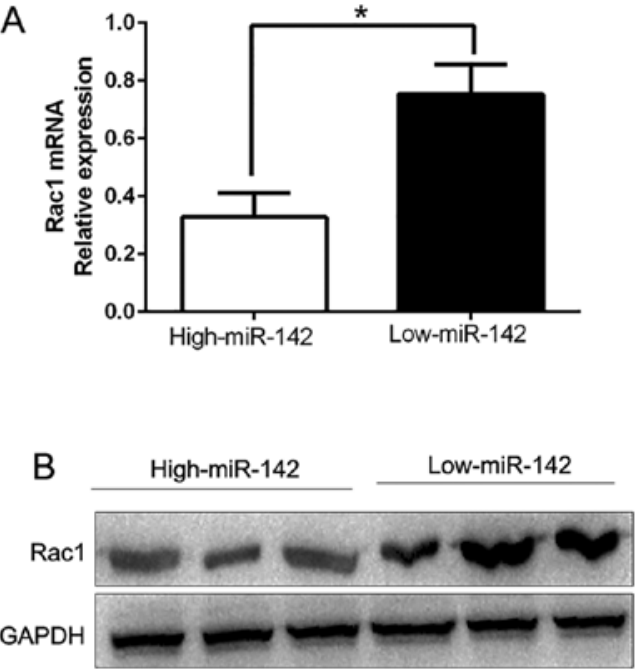

C
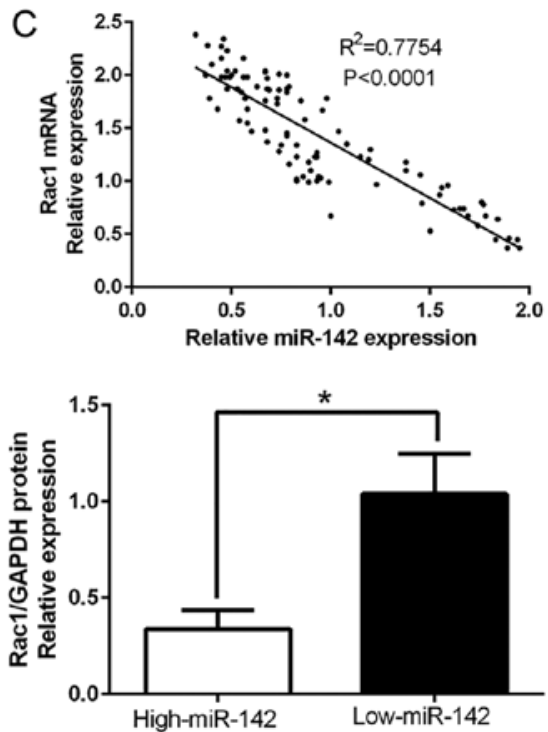

Figure 6. An inverse correlation between miR-142 and Rac1 expression is observed in glioma. (A) The expression of Rac1 mRNA in the miR-142 high-expressing tumors was significantly lower than that in the miR-142 low-expressing tumors. (B) The expression of the Rac1 protein in the miR-142 high-expressing tumors was significantly lower than that in the miR-142 low-expressing tumors. (C) A significant inverse correlation between the mRNA levels of Rac1 and miR-142 was observed in glioma tissues; ${ }^{*} \mathrm{P}<0.05$.

that the 3'-UTR of Rac1 contains a putative binding site for miR-142 (Fig. 5A). A luciferase assay was performed to investigate whether miR-142 directly targets Rac1. Our data demonstrated that ectopic expression of miR-142 induced a clear decrease of the luciferase activity of the wild-type (wt) Rac1 3'-UTR, but did not inhibit the activity of Rac1 with a mutant (mt) 3'-UTR (Fig. 5B; P<0.05). In addition, miR-142 overexpression obviously suppressed Racl mRNA (Fig. 5C; $\mathrm{P}<0.05$ ) and protein (Fig. 5D; $\mathrm{P}<0.05$ ) expression in $\mathrm{U} 87$ and U251 cells. These results indicated that miR-142 directly targeted Rac1 in glioma cells through interaction with its 3'-UTR.

Racl levels are inversely correlated with miR-142 levels in glioma tissues. To further confirm the aforementioned findings, we detected the expression of Rac1 in human glioma tissues. Our data demonstrated that Rac1 mRNA and protein levels were significantly lower in the high miR-142 expression group than that in the low miR-142 expression group in glioma ( $\mathrm{P}<0.05$; Fig. $6 \mathrm{~A}$ and $\mathrm{B})$. In addition, the results revealed that the mRNA level of Racl in glioma tissues was inversely correlated with miR-142 expression $\left(\mathrm{R}^{2}=0.7754\right.$; $\mathrm{P}<0.0001$; Fig. 6C). In conclusion, these data revealed that Rac1 was a direct downstream target of miR-142 in glioma.

Overexpression of Racl expression counteracted the suppressive effects of miR-142 expression. To further confirm whether Racl was involved in miR-142-mediated inhibition of migration, invasion and invasion-related genes, we restored Rac1 expression in U87-miR-142 and U251-miR-142 cells by transfecting Rac1 expression plasmid ( $\mathrm{P}<0.05$; Fig. 7A). The results revealed that Racl overexpression promoted cell migration $(\mathrm{P}<0.05$; Fig. 7B), invasion $(\mathrm{P}<0.05$; Fig. 7C) and invasion-related MMP expression ( $\mathrm{P}<0.05$; Fig. 7D). These data demonstrated that Rac1 is a downstream mediator in the function of miR-142 in glioma.

\section{Discussion}

Recently, accumulating evidence has confirmed that aberrant miRNAs are involved in the initiation, development and progression of human malignancies via modulation of their targeting oncogenes or tumor-suppressor genes, which could potentially serve as biomarkers for the prediction and prognosis of diverse cancers, including glioma. Therefore, identification of cancer-specific miRNAs is critical for elucidating their molecular mechanism and vital for developing novel therapeutic targets. In previous studies, miRNA-142 negatively regulated canonical Wnt/ $\beta$-catenin signaling, which plays pivotal roles in cancer progression (29). Schwickert et al demonstrated that miRNA-142 inhibits breast cancer cell invasiveness by synchronous targeting of WASL, integrin $\alpha \mathrm{V}$ and additional cytoskeletal elements (30). miR-142 functions as a potential tumor suppressor directly targeting HMGB1 in non-small cell lung carcinoma (31). However, conversely, miR-142 could promote cell proliferation in nasopharyngeal carcinoma (24). Moreover, miR-142 plays an oncogenic role in cell migration, proliferation and apoptosis in renal cell carcinoma (28). These data indicated that the expression level and biological function of miR-142 was cancer type-dependent.

In the present study, we confirmed that the expression of miR-142 was significantly downregulated in clinical specimens and cell lines. Moreover, our data revealed that decreased expression of miR-142 was associated with advanced WHO grade in glioma. In addition, miR-142 expression was an independent prognostic indicator in the prediction of the 5-year overall survival of glioma patients. Collectively, these data revealed that miR-142 may play a critical role in the development of glioma and the decreased expression of miR-142 confers a worse prognosis and could be identified as a prognostic indicator. Mechanically, overexpression of miR-142 suppressed the cell migration and invasion capacity of glioma U87 and U251 cells. Moreover, miR-142 inhibited 
A
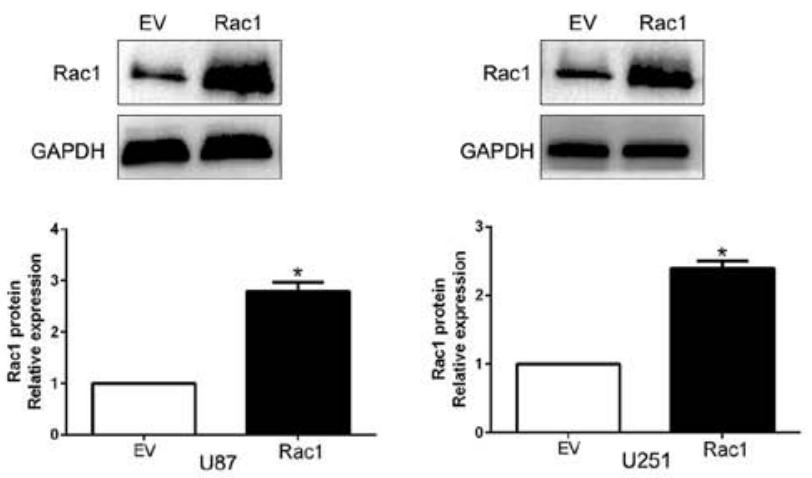

C
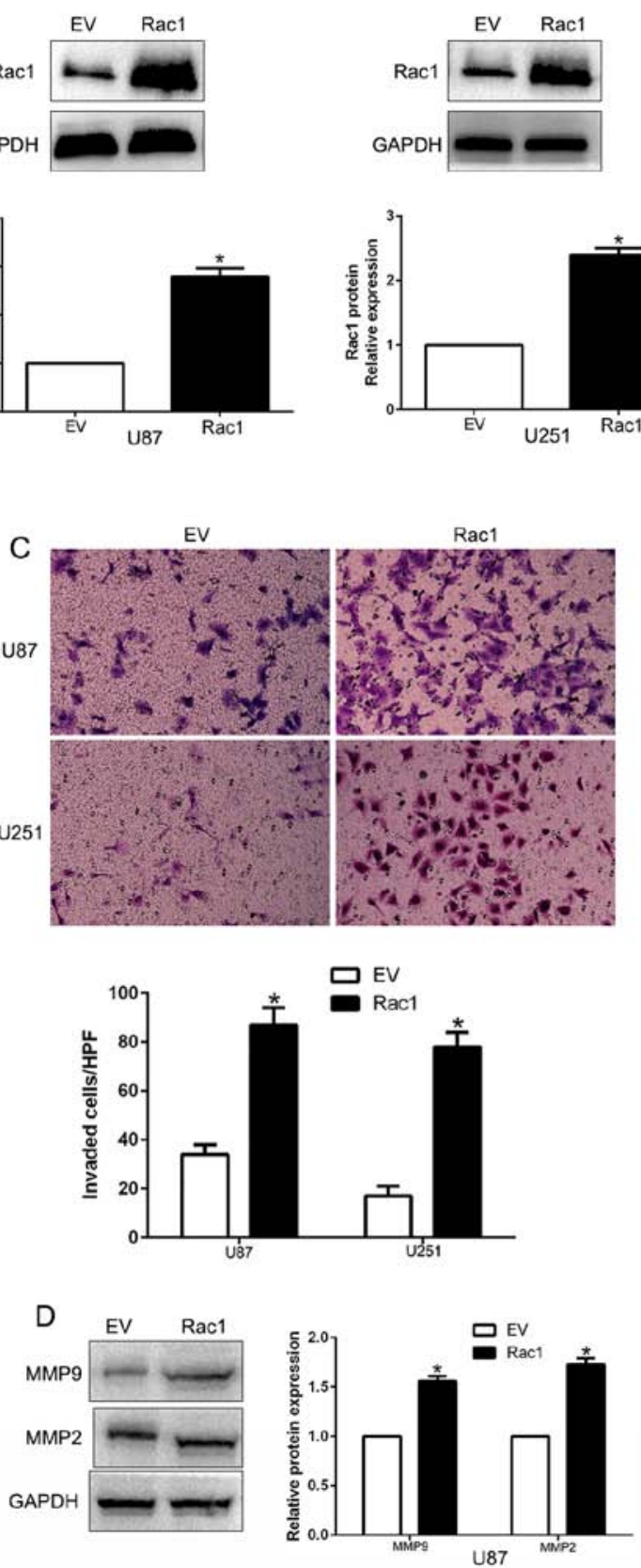

B

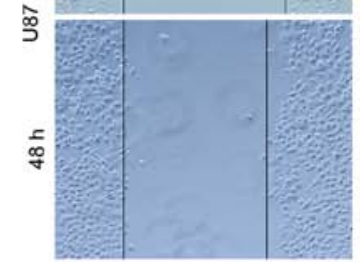

EV
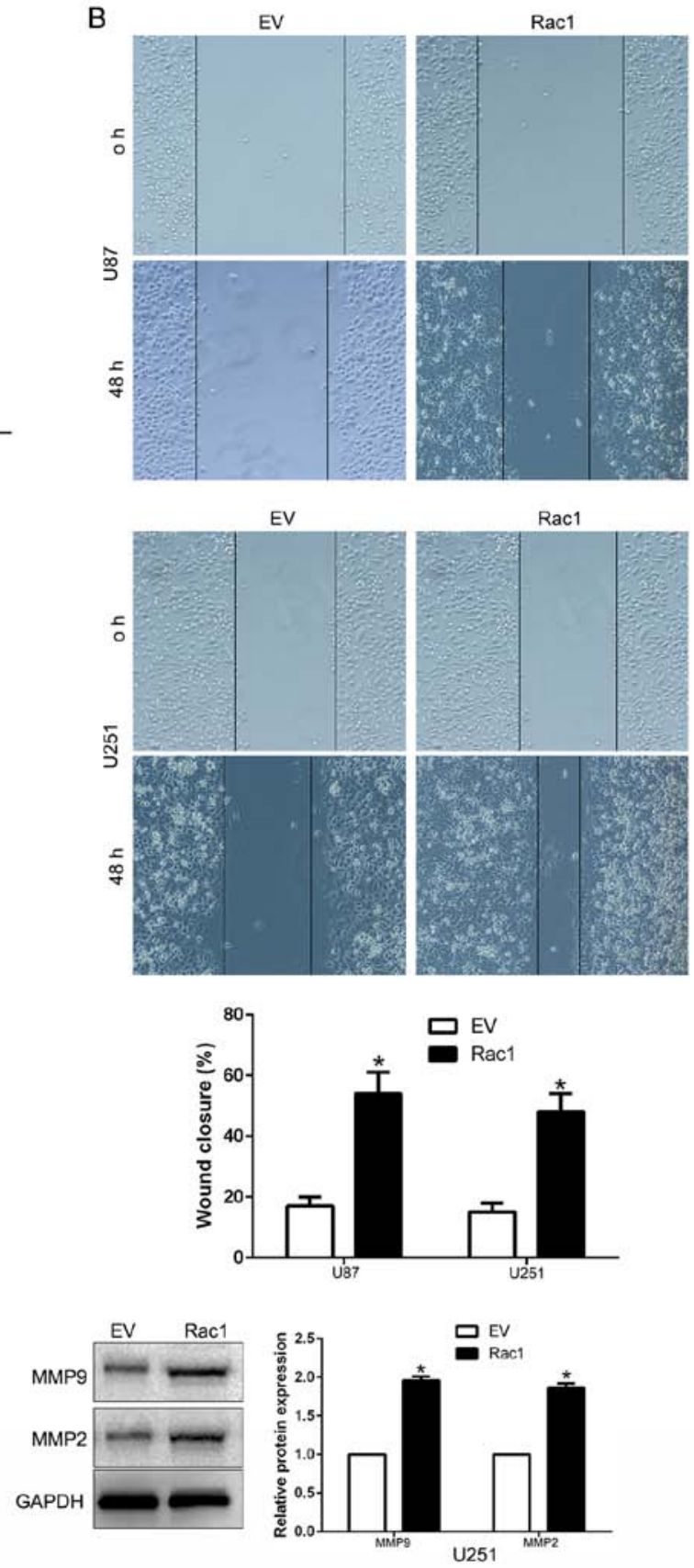

Figure 7. Alterations of Racl partially abolish miR-142-mediated glioma cell migration, invasion and MMP expression. (A) miR-142-overexpressing U87 and U251 cells that were transfected with EV or Rac1 expression plasmid were subjected to western blot analysis to determine the expression of Rac1. (B) Cell migration of the miR-142-overexpressing U87 and U251 cells was promoted by Rac1 overexpression. (C) Ectopic Rac1 expression abrogated the effects of miR-142 overexpression in U87 and U251 cells. (D) Western blot analysis of MMP2 and MMP9 expression in U87 and U251 cells stably expressing miR-142 transduced with Rac1 or control vector. $\mathrm{n}=$ six independent experiments. "P<0.05. EV, empty vector; MMP, matrix metalloproteinase.

the invasion-related MMP gene expression. Furthermore, we demonstrated that Rac1 was a direct target of miR-142 in glioma, which was consistent with studies on hepatocellular carcinoma and osteosarcoma $(18,32)$. We also observed that miR-142 overexpression suppressed the expression of Rac1 mRNA and protein. Furthermore, we found an inverse correlation between miR-142 expression and Rac1 in glioma tissues. Additionally, the effects of miR-142 alteration on cell migration and invasion of glioma cells were abolished by Rac1 modulation. Thus, these results indicated that miR-142 inhibited migration and invasion in glioma cells by directly blocking the Racl pathway.
Emerging evidence has revealed that Rac1-dependent signaling is essential for tumor initiation, development and progression (33). The expression of Rac1 has been demonstrated to be much higher in several human cancers, including glioma (34). The high expression of Rac1 induced glioma cell proliferation and inhibited apoptosis. Moreover, previous studies confirmed that Rac1 could promote migration and invasion by inducing epithelial-to-mesenchymal transition (EMT) and MMP expression (35), which have been identified as key regulators in the invasion and metastasis of diverse cancers. In the present study, we found miR-142 inhibited MMP expression by targeting Rac1. Rac1 restoration abrogated the suppressive 
effect of miR-142. Collectively, these data demonstrated the suppressive effect of miR-142 was mediated by targeting Rac1 to inhibit MMP expression in glioma.

In conclusion, we demonstrated that miR-142 was downregulated in glioma tissues and cell lines, and its decreased expression was associated with advanced clinicopathological features. Furthermore, we confirmed that miR-142 inhibited cell migration, invasion and MMP expression by inhibiting Racl. These results revealed that miR-142 is a potential metastasis-associated tumor suppressor in glioma. Collectively, decreased miR-142 expression may play an important role in tumor metastasis and may be a novel prognostic factor and potential therapeutic target for glioma.

\section{Acknowledgements}

The present study was supported by a grant from the National Natural Scientific Foundation of China (no. 81403243).

\section{References}

1. Vredenburgh JJ, Desjardins A, Reardon DA and Friedman HS: Experience with irinotecan for the treatment of malignant glioma. Neuro Oncol 11: 80-91, 2009.

2. Xie Q, Mittal S and Berens ME: Targeting adaptive glioblastoma: An overview of proliferation and invasion. Neuro Oncol 16: 1575-1584, 2014.

3. Preusser M, Haberler C and Hainfellner JA: Malignant glioma: Neuropathology and neurobiology. Wien Med Wochenschr 156: 332-337, 2006.

4. Vigneswaran K, Neill S and Hadjipanayis CG: Beyond the World Health Organization grading of infiltrating gliomas: Advances in the molecular genetics of glioma classification. Ann Transl Med 3: 95, 2015.

5. Cohen AL and Colman H: Glioma biology and molecular markers. Cancer Treat Res 163: 15-30, 2015.

6. Stupp R, Mason WP, van den Bent MJ, Weller M, Fisher B, Taphoorn MJ, Belanger K, Brandes AA, Marosi C, Bogdahn U, et al; European Organisation for Research and Treatment of Cancer Brain Tumor and Radiotherapy Groups; National Cancer Institute of Canada Clinical Trials Group: Radiotherapy plus concomitant and adjuvant temozolomide for glioblastoma. $\mathrm{N}$ Engl J Med 352: 987-996, 2005.

7. Davis FG and McCarthy BJ: Current epidemiological trends and surveillance issues in brain tumors. Expert Rev Anticancer Ther 1: 395-401, 2001.

8. Makeyev EV and Maniatis T: Multilevel regulation of gene expression by microRNAs. Science 319: 1789-1790, 2008.

9. Bartels CL and Tsongalis GJ: MicroRNAs: Novel biomarkers for human cancer. Clin Chem 55: 623-631, 2009.

10. Esquela-Kerscher A and Slack FJ: Oncomirs - microRNAs with a role in cancer. Nat Rev Cancer 6: 259-269, 2006.

11. Liu Z, Dou C, Yao B, Xu M, Ding L, Wang Y, Jia Y, Li Q, Zhang $\mathrm{H}, \mathrm{Tu} \mathrm{K}$, et al: Methylation-mediated repression of microRNA-129-2 suppresses cell aggressiveness by inhibiting high mobility group box 1 in human hepatocellular carcinoma. Oncotarget 7: 36909-36923, 2016.

12. Wu J, Cui H, Zhu Z and Wang L: MicroRNA-200b-3p suppresses epithelial-mesenchymal transition and inhibits tumor growth of glioma through down-regulation of ERK5. Biochem Biophys Res Commun 478: 1158-1164, 2016.

13. Deng B, Zhang Y, Zhang S, Wen F, Miao Y and Guo K: MicroRNA-142-3p inhibits cell proliferation and invasion of cervical cancer cells by targeting FZD7. Tumour Biol 36: 8065-8073, 2015.

14. Malzkorn B, Wolter M, Liesenberg F, Grzendowski M, Stühler K, Meyer HE and Reifenberger G: Identification and functional characterization of microRNAs involved in the malignant progression of gliomas. Brain Pathol 20: 539-550, 2010.

15. Cao XC, Yu Y, Hou LK, Sun XH, Ge J, Zhang B and Wang X: miR-142-3p inhibits cancer cell proliferation by targeting CDC25C. Cell Prolif 49: 58-68, 2016.
16. Pan D, Du Y, Ren Z, Chen Y, Li X, Wang J and Hu B: Radiation induces premature chromatid separation via the miR-142-3p/Bod1 pathway in carcinoma cells. Oncotarget 7: 60432-60445, 2016.

17. Ma Z, Liu T, Huang W, Liu H, Zhang HM, Li Q, Chen Z and Guo AY: MicroRNA regulatory pathway analysis identifies miR-142-5p as a negative regulator of TGF- $\beta$ pathway via targeting SMAD3. Oncotarget 7: 71504-71513, 2016.

18. Zheng Z, Ding M, Ni J, Song D, Huang J and Wang J: miR-142 acts as a tumor suppressor in osteosarcoma cell lines by targeting Rac1. Oncol Rep 33: 1291-1299, 2015.

19. Su YH, Zhou Z, Yang KP, Wang XG, Zhu Y and Fa XE: MIR-142-5p and miR-9 may be involved in squamous lung cancer by regulating cell cycle related genes. Eur Rev Med Pharmacol Sci 17: 3213-3220, 2013.

20. Yin Y, Song M, Gu B, Qi X, Hu Y, Feng Y, Liu H, Zhou L, Bian Z, Zhang J, et al: Systematic analysis of key miRNAs and related signaling pathways in colorectal tumorigenesis. Gene 578: $177-184,2016$.

21. Shen WW, Zeng Z, Zhu WX and Fu GH: MiR-142-3p functions as a tumor suppressor by targeting CD133, ABCG2, and Lgr5 in colon cancer cells. J Mol Med 91: 989-1000, 2013.

22. Xu G, Wang J, Jia Y, Shen F, Han W and Kang Y: MiR-142-3p functions as a potential tumor suppressor in human osteosarcoma by targeting HMGA1. Cell Physiol Biochem 33: 1329-1339, 2014.

23. Kaduthanam S, Gade S, Meister M, Brase JC, Johannes M, Dienemann H, Warth A, Schnabel PA, Herth FJ, Sültmann H, et al: Serum miR-142-3p is associated with early relapse in operable lung adenocarcinoma patients. Lung Cancer 80: 223-227, 2013.

24. Qi X, Li J, Zhou C, Lv C and Tian M: MiR-142-3p suppresses SOCS6 expression and promotes cell proliferation in nasopharyngeal carcinoma. Cell Physiol Biochem 36: 1743-1752, 2015.

25. Pelloni M, Coltrinari G, Paoli D, Pallotti F, Lombardo F, Lenzi A and Gandini L: Differential expression of miRNAs in the seminal plasma and serum of testicular cancer patients. Endocrine: Oct 28, 2016 (Epub ahead of print). https://doi.org/10.1007/ s12020-016-1150-z.

26. Summerer I, Unger K, Braselmann H, Schuettrumpf L, Maihoefer C, Baumeister P, Kirchner T, Niyazi M, Sage E, Specht HM, et al: Circulating microRNAs as prognostic therapy biomarkers in head and neck cancer patients. Br J Cancer 113: 76-82, 2015.

27. Lin RJ, Xiao DW, Liao LD, Chen T, Xie ZF, Huang WZ, Wang WS, Jiang TF, Wu BL, Li EM, et al: MiR-142-3p as a potential prognostic biomarker for esophageal squamous cell carcinoma. J Surg Oncol 105: 175-182, 2012.

28. Li Y, Chen D, Jin LU, Liu J, Li Y, Su Z, Qi Z, Shi M, Jiang Z, Yang S, et al: Oncogenic microRNA-142-3p is associated with cellular migration, proliferation and apoptosis in renal cell carcinoma. Oncol Lett 11: 1235-1241, 2016.

29. Isobe T, Hisamori S, Hogan DJ, Zabala M, Hendrickson DG, Dalerba P, Cai S1, Scheeren F, Kuo AH1, Sikandar SS, et al: miR-142 regulates the tumorigenicity of human breast cancer stem cells through the canonical WNT signaling pathway. Elife 3: doi: 10.7554/eLife.01977, 2014.

30. Schwickert A, Weghake E, Brüggemann K, Engbers A, Brinkmann BF, Kemper B, Seggewiß J, Stock C, Ebnet K, Kiesel L, et al: microRNA miR-142-3p inhibits breast cancer cell invasiveness by synchronous targeting of WASL, integrin alpha V, and additional cytoskeletal elements. PLoS One 10: e0143993, 2015.

31. Xiao P and Liu WL: MiR-142-3p functions as a potential tumor suppressor directly targeting HMGB1 in non-small-cell lung carcinoma. Int J Clin Exp Pathol 8: 10800-10807, 2015.

32. Wu L, Cai C, Wang X, Liu M,Li X and Tang H: MicroRNA-142-3p, a new regulator of $\mathrm{RAC1}$, suppresses the migration and invasion of hepatocellular carcinoma cells. FEBS Lett 585: 1322-1330, 2011.

33. Yoon CH, Hyun KH, Kim RK, Lee H, Lim EJ, Chung HY, An S, Park MJ, Suh Y, Kim MJ, et al: The small GTPase Racl is involved in the maintenance of stemness and malignancies in glioma stem-like cells. FEBS Lett 585: 2331-2338, 2011.

34. Zhong J, Bach CT, Shum MS and O'Neill GM: NEDD9 regulates 3D migratory activity independent of the Racl morphology switch in glioma and neuroblastoma. Mol Cancer Res 12: 264-273, 2014.

35. Man J, Shoemake J, Zhou W, Fang X, Wu Q, Rizzo A, Prayson R, Bao S, Rich JN and Yu JS: Sema3C promotes the survival and tumorigenicity of glioma stem cells through Rac1 activation. Cell Rep 9: 1812-1826, 2014. 